2. Janzen, F. J. \& Paukstis, G. L Q. Rev Biol. 66, 149-179 (1991).

3. Cree, A., Thompson, M. B. \& Daugherty, C. H. Nature 375, 543 (1995).

4. Ciofi, C. \& Swingland, I. R. App. Anim. Behav. Sci. 51, 251-265 (1997).

5. Cogger, H. G. Reptiles and Amphibians of Australia 3rd edn (Reed, Sydney, 1986).

6. Harlow, P. S. Herpet. Rev. 27, 71-72 (1996).

7. Yntema, C. L. J. Morphol. 167, 297-304 (1981).

8. Yntema, C. L. \& Mrosovsky, N. Herpetologica 36, 33-36 (1980)

9. Olsson, M. \& Shine, R. J. Evol. Biol. 14, 120-128 (2001).

10. Schwarzkopf, L. \& Shine, R. Oecologia 88, 562-569 (1991).

11. Janzen, F. J. Proc. Natl Acad. Sci. USA 91, 7487-7490 (1994).

12. Whetton, P. H. et al. Climate Res. 16, 181-201 (2001).

\section{Ancient chronology}

\section{Astronomical orientation of the pyramids}

pence speculates that Egypt's pyramid builders found true north by using a plumb line: when the stars Kochab and Mizar were seen on the same vertical, one was facing north ${ }^{1}$. As evidence in support of this hypothesis, she points to the proposed interstar-line precession past the north celestial pole at a rate of $27^{\prime}$ per century (cy). We argue that a mathematical error affects this result, which when corrected points more strongly to a different pair of stars. This suggests that the conventional ancient chronology, instead of being compressed, may actually have to be expanded slightly.

The elementary error here is that the interstar-line drift of $27^{\prime} \mathrm{cy}^{-1}$ occurs at an altitude of $30^{\circ}$ (Giza's latitude), but to apply this drift to ground orientation, one must divide by cosine $30^{\circ}$. So, on the ground, $31^{\prime}$ $\mathrm{cy}^{-1}$ is the actual misorientation rate. Thus, the actual drift rate is significantly greater than Spence's empirical rate of orientation change of the pyramids themselves (the slope of line $\boldsymbol{a}$ in Fig. 4 of ref. 1).

When the pyramids were built, only two stars brighter than fifth magnitude lay anywhere near the pole: Thuban (3.65 mag, $1.5^{\circ}$ distant) and the irregularly variable star 10 Draconis (4.5-5.0 mag). In $2627 \mathrm{BC}$, the pole was equidistant $\left(1^{\circ}\right)$ from each star, so the pole was the obtuse apex of a squat isosceles triangle formed between itself and the two stars. When both stars were at the same altitude, north was the direction bisecting them. (For more than a millennium after $2627 \mathrm{BC}$, there was no star brighter than 10 Draconis nearer to the celestial pole.)

Among several mechanical methods, north could have been determined in the dark by sighting the two horizontal stars simultaneously against a pointed post, the pyramidal top of an obelisk, or any similar object; when the observer can eclipse both stars simultaneously on opposite sides of the peak, the line from the observer to the peak points northwards. This simultaneous-eclipse method does not require the post or obelisk to be illuminated, making it simpler than Spence's plumb-line method; there is no easy way to see a plumb line at night while retaining the observer's nightvision acuity.

Although 10 Draconis is barely visible under modern industrial skies, it is recorded in all four large preclassical naked-eye star catalogues: Hipparchos, 128 BC; Ulugh Beg, AD 1437; Tycho, AD 1601; and Hevelius, AD 1661. Spence's Kochab and Mizar are indeed brighter than Thuban, but the eyeprecision she assumes implies that the Kochab-Mizar line will confusingly pass into detectable and uncentred non-verticality in a matter of a few (perhaps ten) seconds. (Spence's suggestions of 5-year or 1-2-year precision for dating the pyramids imply a surveying precision of about $1^{\prime}$.) So Spence's method, although possible, would require agile quickness. In contrast, the midpoint between Thuban and 10 Draconis gives a ground orientation within $1^{\prime}$ of true north for over 5 minutes on either side of its transit. The very slow motion of these stars (and the small size of any potential orientation error from their use) is due to their close proximity to the celestial pole.

The precision of raw, naked-eye stellar observations can be significantly better than $3^{\prime}$, but we justify the utility of our two stars by reference to the scrupulous naked-eye catalogues of Tycho and Hevelius ${ }^{2,3}$. Tycho's raw data survive for both stars ${ }^{4}$, eight observations in all: r.m.s. errors are $2^{\prime}$ for Thuban and $3^{\prime}$ for 10 Draconis. In Hevelius' catalogue, the equatorial coordinates of Thuban and 10 Draconis (his Draco stars 8 and 32) have great-circle errors of $1^{\prime}$ and $0^{\prime}$, respectively. Thus, the dimness of 10 Draconis was in itself no barrier to accurate measurement of its position in pre-industrial times, and such precision could easily be replicated for an azimuth observation, even using simple instruments, by positioning the observer's eye at a large enough distance from the eclipsing post.

In $2627 \mathrm{BC}$, the misorientation associated with our obvious and straightforward method was null but precessionally increasing at $27.4^{\prime} \mathrm{cy}^{-1}$ in azimuth, which matches Spence's $28^{\prime} \mathrm{cy}^{-1}$ empirical rate much more closely than her Mizar-Kochab method $\left(31^{\prime} \mathrm{cy}^{-1}\right)$. This implies dates of $2638 \mathrm{BC}$ for Khufu's pyramid and 2607 BC for Khafre's. (Error estimates could be 2-10 years, depending on assumptions regarding the builders' craftmanship.) These dates are a few decades outside the conventional ranges Spence cites 5 . But our implied date for the ascension of Khufu (2640 BC) is twice as near to a conventional boundary as Spence's 2480 BC (Table 1 in ref. 1). Back-disparity makes more sense than Spence's very forward dates, when current orthodoxy is based on king lists that are "seldom complete"1.

It seems odd that either method would have been used before the time when it was correct. Because the best pyramid orienta- tions occur for the two greatest pyramids, this could simply indicate that engineering science peaked at the time of KhufuKhafre. Thuban passed within $0.1^{\circ}$ of the pole in $2800 \mathrm{BC}$, a chance event that may have stimulated the historical flowering of celestially based surveying, which was unquestionably used for the pyramids built soon after that at Giza. A stellar explanation of the Giza pyramids' location (in latitude) has already been proposed ${ }^{6}$.

Latitude (but not orientation) could be found in a single night near the time of the winter solstice anywhere close to $2600 \mathrm{BC}$ by bisecting Thuban's circumpolar semicircle, because this star was within $10^{\circ}$ of the equinoctial colure for centuries after 2700 BC. Because 10 Draconis was within $10^{\circ}$ of the solstitial colure for three decades either side of $2613 \mathrm{BC}$, on the same or any neighbouring night, orientation might also have been found by bisecting the circumpolar arc of 10 Draconis. Although twilight would have cut the arc to slightly less than $180^{\circ}$, this still would have been adequate for the purpose. By coincidence, both orientation methods that depended on 10 Draconis were most accurate at virtually the same time: 2627 BC for the Spence interstar method applied to Thuban and 10 Draconis, and $2613 \mathrm{BC}$ for the 10 Draconis arc method.

Before Spence's proposal ${ }^{1}$, a possible connection was suggested ${ }^{7}$ between precession and the pyramids' misorientation, but the horizon-based observations proposed would be too prone to difficulties with high refraction, uneven topography, dip and atmospheric extinction to be practical. So, despite a few disagreements, we welcome Spence's creativity in pointing out the possibilities of orienting the pyramids by observing northern stars higher in the sky and near to the meridian, which doubly minimizes corruption by refraction.

Dennis Rawlins ${ }^{\star}$, Keith Pickering $\dagger$

${ }^{\star}$ DIO, The International Journal of Scientific

History, Box 19935, Baltimore,

Maryland 21211-0935, USA

$\dagger$ Analysts International Corporation, 3601 West

76th Street, Minneapolis, Minnesota 55435, USA

e-mail:keithp@minn.net

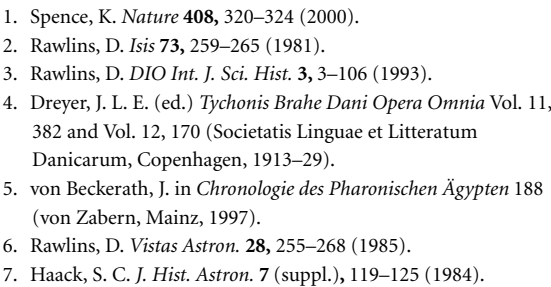

Spence replies - Rawlins and Pickering have correctly identified an error: I should indeed have divided the calculated figures for the distance of the line between $\beta$ Ursae Minoris and $\zeta$ Ursae Majoris from the pole 
by cosine $30^{\circ}$ to convert the rate of drift (which is correct) into azimuth. However, this error does not invalidate my method ${ }^{1}$ : the increase in the slope is small and the revised gradient fits well with the data originally presented (Fig. 1). The decision to discard the alternative pair of stars first considered is also now more convincing, as their revised drift is around 37 $\mathrm{cy}^{-1}$, which cannot be accommodated by the archaeological data. The revision therefore does not affect my results ${ }^{1}$, apart from being more compatible with a shorter reign for Snofru.

Rawlins and Pickering also question whether orientations could be achieved during the short period when the two stars are in simultaneous transit. This would not be a problem as, assuming that the plumb line was hung from a frame, the sighting device could be adjusted to keep the plumb line equidistant between the two stars for several minutes as they came into simultaneous transit. The alignment could also have been checked on successive nights to ensure that it was exact. The fact that this method has a short and clearly defined period (the stars move rapidly out of plumb in opposite directions), within which the alignment is established, is one of the reasons why it is such an accurate method of orientation. The stars used are very bright and low light on a plumb line would not have substantially affected visibility.

I find the alternative method proposed by Rawlins and Pickering unconvincing. 10 Draconis is very faint, barely visible to the naked eye on a clear, dark night. The fact that a star appears in historical star catalogues from $128 \mathrm{BC}$ onwards does not mean that it was used or even recognized by the ancient Egyptians around 2500 BC. These historical star catalogues were compiled within a context of interest in detailed astronomical measurement and recording, for which we have no evidence from ancient Egypt. Establishing when two stars that are so close and faint are exactly horizontal and simultaneously eclipsed would not be easy. In addition, if the Egyptians thought that the pole was equidistant from two stars, both of which are close to the pole and one barely visible, it is unclear why they did not simply use the brighter star ( $\alpha$ Draconis) in a bisection method.

Haack has also suggested a link between the misorientation of pyramids and precession $^{2}$, proposing that the pyramids were oriented towards the rising or setting of a star on the east or west horizon. Variable horizon conditions between sites, and even on the same site (the ground slopes upwards to the west at Giza, precluding observations equivalent to those made to the east), coupled with all the problems involved with observing stars that are close

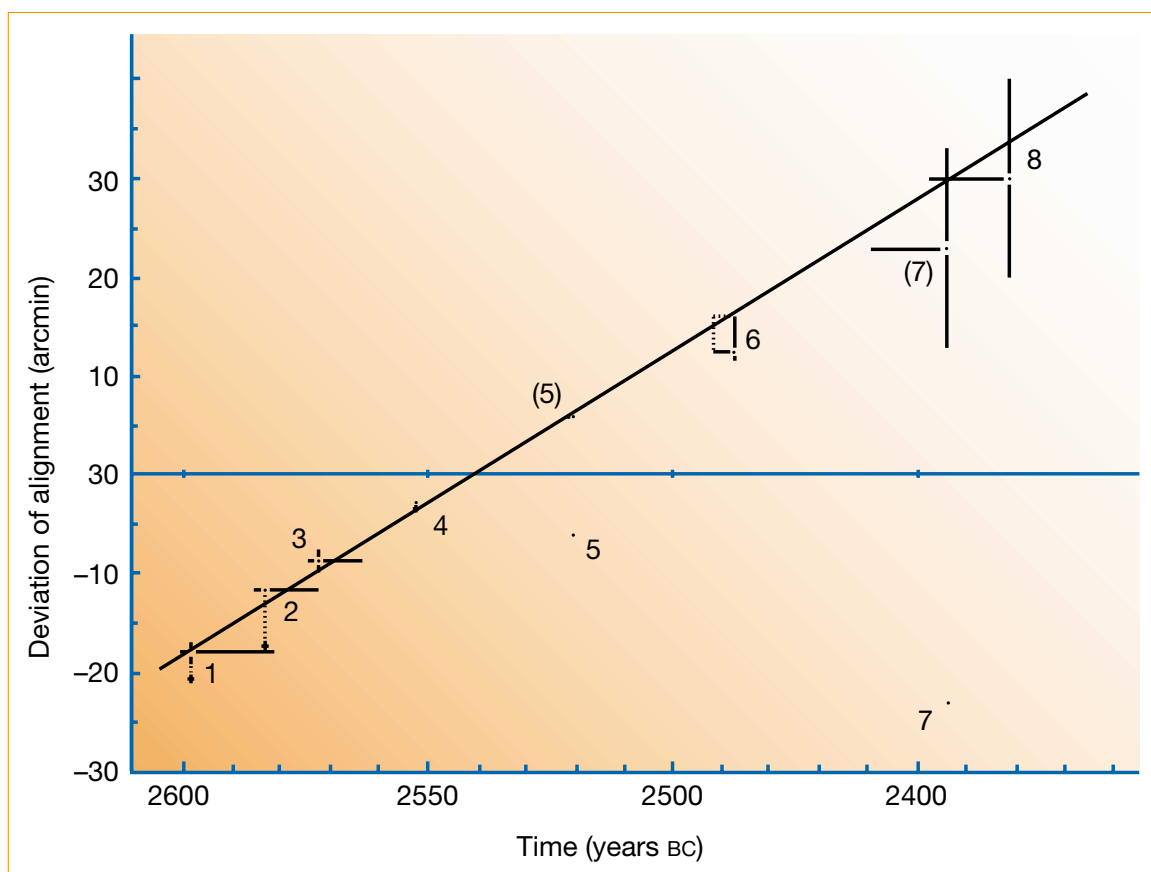

Figure 1 Astronomical modelling of the simultaneous-transit method of orientation. The points are plotted from the data given in Table 1 of ref. 1. The corrected gradient for measurements of orientation using the simultaneous transit of $\beta$ Ursae Minoris and $\zeta$ Ursae Majoris is plotted over the archaeological data. The point at which this line crosses zero can be recalibrated to 2467 BC by astronomical modelling. 1, Meidum; 2, Bent Pyramid; 3, Red Pyramid; 4, Khufu; 5, Khafre; 6, Menkaure; 7, Sahure; 8, Neferirkare. Numbers in parentheses denote points replotted with positive rather than negative values to conform to the dominant trend of the alignments.

to the horizon, make this method untenable. However, as both alternative methods can be modelled to produce results that are comparable with the archaeological data, the context must also be considered. The method I propose and the stars used are consistent with other evidence of Egyptian astronomy at this time.

In Khufu's pyramid, four narrow shafts run north and south at an upward angle from two of the inner chambers. These shafts are probably oriented towards the culminations of important stars $^{3-5}$, although this is not accepted by all Egyptologists. It has been suggested that one of the northern shafts is oriented towards the culmination of $\beta$ Ursae Minoris ${ }^{5}$, which is also one of the stars used in the simultaneous-transit method. Other investigations (T. van Albada and A. Egberts, personal communication) confirm the orientation towards $\beta$ Ursae Minoris and give dates that are compatible with my results. Independent archaeological data for the shafts and the bases suggest that both are aligned towards culminations, that both involve $\beta$ Ursae Minoris and that both require a forward shift of the chronology of the period.

Later pictorial evidence, such as the Senenmut astronomical ceiling, suggests that the Egyptians envisaged a line running between two circumpolar constellations as a method of finding north ${ }^{6}$. One of these constellations is definitely Ursa Major ${ }^{6}$; the other is probably Ursa Minor ${ }^{7}$. Later textual evidence suggests the use of a plumb line and Ursa Major ${ }^{6}$.

The Egyptians were almost certainly unaware that their orientation method was precession-dependent and that it was therefore initially inexact, otherwise they would have used a more accurate bisection method. There is no evidence from any period of their history that the ancient Egyptians measured or understood the concept of latitude, which emerged during Hellenistic times within the context of a far more sophisticated programme of astronomical observation and investigation. Rawlins' speculation ${ }^{8}$ of a stellar explanation of the Giza pyramids' location in terms of latitude is unlikely, as it accounts for the location of only three of the many kings' pyramids in Egypt, and the pyramid of Djedefre, which was constructed between the reigns of Khufu and Khafre, was built at a different site.

\section{Kate Spence}

Faculty of Oriental Studies, University of

Cambridge, Cambridge CB3 9DA, UK

e-mail: kes1004@cam.ac.uk

1. Spence, K. Nature 408, 320-324 (2000).

2. Haack, S. C. J. Hist. Astron. 15 (suppl.), S119-S125 (1984).

3. Badawy, A. Mitteilungen des Instituts für Orientforschung der Deutschen Akademie der Wissenschaften zu Berlin 10, 189-206 (1964).

4. Trimble, V. Mitteilungen des Instituts für Orientforschung der Deutschen Akademie der Wissenschaften zu Berlin 10, 183-187 (1964).

5. Bauval, R. G. \& Gilbert A. G. Disc. Egyptol. 28, 5-13 (1994).

6. Zába, Z. l'orientation astronomique dans l'ancienne Egypte et la

précession de l'axe du monde (Editions de l'Académie Tchécoslovaque des Sciences, Prague, 1953).

7. Davies,V. L. J. Hist. Astron. 16 (suppl.), S102-S104 (1985).

8. Rawlins, D. Vistas Astron. 28, 255-268 (1985). 